\title{
Objetivos y medios idóneos relativos a la dependencia funcional ${ }_{1}$
}

\author{
Demetrio Casado Pérez
}

Seminario de Intervención y Políticas Sociales (SIPOSO)

$<$ demetrio.casado@gmail.com>

\author{
Luisa Fernández Pinar \\ Colegio Oficial de Politólogos y Sociólogos de Madrid
}

\begin{abstract}
Muturreko mendetasun funtzionaleko egoerek sostengu-laguntza gehigarrien premia izaten dute, eta horien gabezia-arriskua betetzea da gizartepolitikaren helburu propioetako bat. Baldintza berekoak dira prebentzioa eta errehabilitazioa, baina ez dira hainbestean aintzako hartzen. Bestetik, Espainian, helburu propioek konpetentzia izaten dute alboko helburuekin, bereziki mendekotasunaren arretaren gizarte- eta ekonomiaitzulkinetan. Arretarako baliabideei dagokionez, Espainian ere erabiltzaileen interesean zentratutako irizpideak aztoratzen ari dira merkatu-hornitzaile eta profesionalen korporazioek egiten ari diren presiogatik. Horrek adierazpen nabaria izan du 2012an buruturiko Mendekotasunaren Legearen erreforman.
\end{abstract}

\section{HITZ-GAKOAK:}

Autonomia pertsonala, zaintzak, mendekotasun funtzionala, prebentzioa, errehabilitazioa, interes korporatiboak.
Las situaciones de dependencia funcional extraordinaria entrañan la demanda de apoyos supletorios, y es un objetivo propio de la política social cubrir el riesgo de carencia de aquéllos. También tienen esa condición la prevención y la rehabilitación, pero son menos valorados. Por otra parte, en España, los objetivos propios sufren la competencia de los colaterales, especialmente el logro de retornos sociales y económicos mediante la atención a la dependencia. En cuanto a los medios de atención, también en España, la presión de proveedores mercantiles y de corporaciones profesionales está alterando la elección de aquéllos según criterios centrados en el interés de los usuarios. Ello ha tenido una manifestación muy ostensible en la reforma de la Ley de Dependencia llevada a cabo en 2012.

\section{Palabras Clave:}

Autonomía personal, cuidados, dependencia funcional, prevención, rehabilitación, intereses corporativos. 


\section{La dependencia funcional}

El objeto específico de este artículo requiere explicitar previamente el ámbito en el que se sitúa, lo que se hará en este primer apartado, siguiendo muy de cerca una publicación de Demetrio Casado (2011a: 55-59).

El término 'dependencia' es un sustantivo verbal abstracto con tantos significados como referentes se le asignen. En el lenguaje de la intervención y las políticas sociales, la palabra 'dependencia' ha venido aplicándose en el pasado, sobre todo, a la pasividad económica asociada, con frecuencia, a la percepción sin contrapartidas de ayudas o prestaciones materiales. Desde hace al menos tres decenios, se ha prodigado el uso de ese término para denotar la vinculación compulsiva a ciertas drogas adictivas. Este artículo no trata de tales clases de la dependencia humana, sino de aquella que consiste en necesidad de ayuda externa extraordinaria y continuada para desenvolverse en la vida común cuando dicha necesidad afecta a personas en edad de autovalerse en ese ámbito; se hace esa salvedad en relación con los niños, cuya subsistencia y desarrollo depende de la crianza y cuidados que les proveen sus padres y otras personas autónomas. A dicha necesidad de ayuda externa extraordinaria se la califica en este artículo de 'funcional', en cuanto que se refiere al funcionamiento humano. Téngase en cuenta que el concepto incluye no sólo lo somático, sino también lo psíquico; y no sólo lo individual, sino igualmente lo social. A propósito de términos, como el contexto indica la clase de dependencia objeto de este texto, en ocasiones se prescindirá del adjetivo.

La dependencia a la que se refiere este artículo puede ser definida en los términos del siguiente texto, procedente de un documento internacional (Consejo de Europa, 1999 [1998]):

La dependencia es un estado en el que se encuentran las personas que, por razones ligadas a la falta o la pérdida de autonomía física, psíquica o intelectual, tienen necesidad de asistencia y/o ayudas importantes a fin de realizar los actos corrientes de la vida diaria.

La dependencia puede afectar a cualquier sector de la población y no sólo a las personas mayores. Si bien es cierto que la dependencia tiende a aumentar con la edad, y que la vejez se caracteriza por aparecer frecuentemente asociada con múltiples problemas de salud, la edad no es el único criterio acerca de este estado.

La dependencia económica no está contemplada en esta recomendación.

La etimología de la voz 'autonomía' apunta a la capacidad de tomar decisiones. Y se emplea con dicha acepción en un texto, relativamente reciente, tan importante como el de la Organización Mundial de la Salud sobre Envejecimiento activo: un marco político, presentado en la Segunda Asamblea Mundial sobre Envejecimiento, celebrada en Madrid en 2002 (OMS, 2002).
La definición de dependencia del Consejo de Europa no toma como referencia la 'autonomía' relativa sólo a la toma de decisiones, sino otra noción más amplia, que incluye también el autovalimiento instrumental. Ésta es la opción que se adopta aquí: la dependencia funcional es la situación opuesta a la autonomía igualmente funcional. El Cuadro 1 puede servir para analizar la estructura del fenómeno en cuestión.

\begin{tabular}{|c|c|c|c|}
\hline \multicolumn{4}{|c|}{ Cuadro 1. Autonomía y dependencia } \\
\hline \multirow{2}{*}{ Capacidades } & \multirow{2}{*}{$\begin{array}{c}\text { Factores } \\
\text { personales }\end{array}$} & \multicolumn{2}{|c|}{ Supuestos extremos } \\
\hline & & Positivo & Negativo \\
\hline $\begin{array}{l}\text { Para tomar } \\
\text { decisiones }\end{array}$ & $\begin{array}{l}\text { Cognitivo } \\
\text { Emocional }\end{array}$ & $\begin{array}{c}\text { Autonomía } \\
\text { mental }\end{array}$ & $\begin{array}{c}\text { Enfermedad } \\
\text { mental } \\
\text { invalidante }\end{array}$ \\
\hline $\begin{array}{l}\text { De desempeño } \\
\text { o realización }\end{array}$ & $\begin{array}{l}\text { Físico } \\
\text { Sensorial } \\
\text { Cognitivo } \\
\text { Emocional } \\
\end{array}$ & $\begin{array}{c}\text { Autonomía de } \\
\text { desempeño }\end{array}$ & $\begin{array}{l}\text { Dependencia } \\
\text { de desempeño }\end{array}$ \\
\hline \multirow[t]{2}{*}{$\begin{array}{l}\text { De decisión y } \\
\text { de desempeño } \\
\text { o realización }\end{array}$} & $\begin{array}{l}\text { Cognitivo } \\
\text { Emocional } \\
\text { Físico } \\
\text { Sensorial }\end{array}$ & $\begin{array}{c}\text { Autonomía } \\
\text { funcional }\end{array}$ & $\begin{array}{c}\text { Dependencia } \\
\text { funcional }\end{array}$ \\
\hline & $\begin{array}{c}\text { Factores } \\
\text { estructurales }\end{array}$ & & \\
\hline $\begin{array}{l}\text { De ejecutar } \\
\text { decisiones }\end{array}$ & Relacional & $\begin{array}{c}\text { Autonomía } \\
\text { o capacidad } \\
\text { jurídica y fáctica }\end{array}$ & $\begin{array}{c}\text { Incapacitación } \\
\text { y } \\
\text { sujeción }\end{array}$ \\
\hline
\end{tabular}

Fuente: Casado (2011a: 58).

La etimología del término 'autonomía' invita a considerar primero aquella acepción de este término que denota capacidad de tomar decisiones -entiéndase, oportunas, al menos para el sujeto y sus relaciones-. Esta capacidad requiere conocimiento y juicio, así como también interés y voluntad -recuérdense al efecto las situaciones de depresión-. También se utiliza la palabra 'autonomía' para indicar la capacidad operativa de realizar actividades, desde las más básicas, como el autocuidado, hasta las más específicas de la condición humana, como el estudio, el trabajo y la participación en la vida social. La dependencia objeto de este artículo incluye también las situaciones de quiebra de esa segunda vertiente de la autonomía. En algunos casos extremos, como ciertas demencias en grados avanzados, la pérdida de autonomía funcional puede afectar al conjunto de factores a asociados a aquélla, lo que supone la dependencia funcional total.

Tal vez sea oportuno aclarar que la dependencia funcional objeto de este artículo no incluye las situaciones impuestas de privación de capacidad para tomar decisiones efectivas (incapacitación jurídica) o de ejecutarlas (contención, prisión).

\section{Objetivos y su elección}

Frente a la discapacidad, especialmente cuando ésta entraña dependencia funcional, el sentido de humanidad viene suscitando el objetivo obvio de 
proveer apoyos supletorios de sus carencias funcionales a las personas afectadas. Pero los avances en el conocimiento aplicable a la salud y al bienestar han posibilitado la adopción de otros objetivos igualmente idóneos, aunque con menor visibilidad social. Mediante otras vías de conocimiento, como la económica, se llega a la formulación de objetivos colaterales, que compiten con los propios.

\subsection{Objetivos propios y colaterales}

Como quiera que la dependencia funcional es una circunstancia derivada de enfermedades agudas o crónicas, o de deficiencias, están indicados para aquélla los objetivos de prevención primaria, secundaria y terciaria de dichas alteraciones de la salud: rehabilitación educacional ocupacional e integración relacional, accesibilidad pro equiparación de oportunidades, y asistencia de mantenimiento (Casado, 2011a: 127-176).

La prevención primaria en salud persigue evitar la aparición de defectos congénitos, enfermedades y traumatismos. Una parte de tales alteraciones pueden generar limitaciones graves que supongan dependencia funcional, por lo que la prevención primaria aludida es un objetivo propio del afrontamiento de la dependencia. Se trata de una meta que interesa a todo el ámbito subjetivo de la salud y, de modo especial, a los individuos y a las situaciones del ciclo vital más vulnerables: embriones y fetos, primera infancia, envejecimiento.

La prevención secundaria está indicada ante alteraciones de salud emergentes. Persigue reducir su gravedad o sus efectos duraderos. Aparentemente, este objetivo coincide con el de curación de enfermedades y heridas, pero presenta la peculiaridad de que se aplica con anticipación, como en los nacidos prematuros y de bajo peso o, yendo al otro extremo del ciclo vital, en los maduros o longevos con tensión arterial elevada o hiperlipemia.

La prevención terciaria tiene su ocasión tras manifestarse plenamente una enfermedad u ocurrir un traumatismo. Debe estar vinculada a los objetivos de curación y de rehabilitación médica.

La prevención secundaria y, sobre todo, la terciaria incluyen la rehabilitación médica. Pero hemos de reseñar también el objetivo conexo de la rehabilitación educativa, ocupacional y laboral, y de la integración económico-social. Los accidentes causantes de lesiones medulares generan pérdidas funcionales de gravedad variable; el objetivo de la rehabilitación médica apunta a reducir su intensidad -coincide, pues, con la prevención terciaria-. La otra rehabilitación persigue obtener capacidades para el desempeño por modos alternativos de la vida personal, como la adquisición de formación y destreza para trabajos que no requieran desplazamientos frecuentes, fuerza física o manipulación fina. Este ejemplo sirve para mostrar que el objetivo de esta segunda rehabilitación debe asociarse a opciones determinadas del de integración socioeconómica.

Una tercera meta proactiva es la de accesibilidad. Junto a la prevención y la rehabilitación, en 1983, las Naciones Unidas, en su Programa de Acción Mundial para las Personas con Discapacidad, propuso el ideal de igualdad de oportunidades, que definió así:

Equiparación de oportunidades significa el proceso mediante el cual el sistema general de la sociedad -tal como el medio físico y cultural, la vivienda y el transporte, los servicios sociales y sanitarios, las oportunidades de educación y trabajo, la vida cultural y social, incluidas las instalaciones deportivas y de recreo- se hace accesible para todos (párrafo 12).

Como indica el texto trascrito, el objetivo de accesibilidad no se plantea sólo en relación al medio físico, sino a otros ámbitos de desenvolvimiento personal y participación social.

Un cuarto objetivo concerniente a la dependencia funcional es el de la asistencia de mantenimiento -objetivo omitido, por cierto, en el documento de las Naciones Unidas recién citado-. Como quedó dicho, la dependencia entraña la demanda de apoyos supletorios de las carencias funcionales. El grueso de tales carencias afecta a actividades operacionales y mentales propias del desenvolvimiento personal, pero en algunos casos afecta a las funciones biológicas vitales, desde la respiración hasta la conciencia.

Para preparar la que vendría a ser la Ley de Promoción de la Autonomía Personal y de Atención a las personas en situación de dependencia (en adelante, LAAD), se elaboró un libro blanco (Ministerio de Trabajo y Asuntos Sociales, 2005), que aborda los objetivos propios reseñados, con especial atención al de asistencia de mantenimiento; pero propone también otros. El capítulo $X$ versa sobre la "generación de empleo y retornos económicos y sociales derivados de la implantación del Sistema Nacional de Dependencia". Se trata de objetivos obviamente legítimos, pero de carácter colateral, no centrados en las personas destinatarias de la LAAD. Como se verá, no se incluyeron en el libro blanco sólo por criterio académico.

\subsection{Cuestiones relativas a la elección de objetivos}

Los objetivos propios están avalados por la racionalidad y por gozar de amplio apoyo experto en el ámbito sanitario. Pero su elección, en la práctica, choca con impedimentos conceptuales y prácticos.

En el uso internacional, la noción de cuidados de larga duración abarca los destinados a las situaciones de dependencia. Cabría incluir los que apuntan a prevenirlas, pero al menos algunas visiones de dicha noción no apuntan a las metas más proactivas de las anteriormente expuestas. Así, en la definición adop- 
tada en un documento de consenso del Programa sobre Envejecimiento y Salud de la OMS (2000: 8), se toman como referencia las situaciones de dependencia establecidas:

Los cuidados de larga duración son el sistema de actividades llevadas a cabo por cuidadores informales (familiares, amigos o vecinos) o profesionales (sanitarios, sociales u otros), o ambos, para conseguir que una persona que no sea totalmente capaz de cuidar de sí misma mantenga la mejor calidad de vida posible, de acuerdo con sus preferencias individuales, con el mayor grado posible de independencia, autonomía, participación, realización personal y dignidad humana.

Leichesenrnig, Billings y Nies, en un trabajo reciente sobre cuidados de larga duración (2013: 330), ponderan la importancia, sobre todo de cara al futuro, de la prevención y la rehabilitación en el sector de la población de mayor edad, a la vez que observan una situación de desarrollo moderado de la práctica de dicha clase de cuidados.

Aparte de otras circunstancias, la complejidad y diversidad del conjunto de los objetivos concernientes a la dependencia parecen dificultar que puedan ser abarcados de modo cabal por un solo sistema o rama político-social, como la Seguridad Social, la sanidad o la relativa a la dependencia. Veamos el caso de esta última en España.

En el título del citado libro blanco de la LAAD (Ministerio de Trabajo y Asuntos Sociales, 2005), sólo está indicado el objetivo de atención. La locución 'autonomía personal' aparece referida a un servicio del catalogo que se propone, no como un objetivo (ídem: 753); servicio que se presenta como de "ayuda personal” (ídem: 754). El objetivo de promoción de la autonomía personal fue incorporado por el Ministerio de Trabajo y Asuntos Sociales al anteproyecto de la norma. Tal objetivo podría haber incluido todos los de carácter preventivo y rehabilitador reseñados anteriormente, pero no fue así. Dejando aparte otras consideraciones, anula esa posibilidad la circunstancia de que la acción de la LAAD se limita a las personas a las que se les ha reconocido la afectación de dependencia (art. 5.1.a).

Los objetivos de carácter social y económico colaterales a los propios del afrontamiento de la dependencia deben subordinarse a éstos. Pero, como se verá más adelante, no siempre se hace así. En este caso, se produce una grave vulneración del principio ético que prescribe no tomar a las personas como medio. Se trata del imperativo categórico de Kant, en su fórmula del fin en sí mismo: "obra de modo que uses la humanidad, tanto en tu persona como en la persona de cualquier otro, siempre como un fin al mismo tiempo y nunca solamente como un medio en sí mismo" (1972: 44 y 45).

\section{Medios satisfactores, agentes y modos de provisión}

Para la procura de los objetivos propios del afrontamiento de la dependencia funcional, se dispone de un amplio y diverso arsenal de medios, tanto del ámbito personal y familiar, como de las diferentes ramas del bienestar-especialmente las de aseguramiento de rentas, empleo, urbanismo y vivienda, sanidad y servicios sociales- (Casado, 2006-2007). También son varios los agentes provisores de medios y los modos de provisión. La limitación del espacio disponible en este artículo hace imposible la reseña general de ese complejo, de modo que este apartado se referirá sólo, a modo de muestra, a los medios del primero y del último de los objetivos presentados anteriormente, es decir, a la prevención primaria y la asistencia de mantenimiento.

\subsection{Prevención primaria}

El Cuadro 2 muestra, por medio de ejemplos, que la prevención primaria de enfermedades, traumatismos y deficiencias, que en algunos casos pueden generar situaciones de dependencia, se realiza por muy diversas clases de medios.

\begin{tabular}{|l|l|}
\hline \multicolumn{2}{|c|}{ Cuadro 2. Clases de intervenciones en prevención primaria } \\
\hline Intervenciones individuales & Acciones de alcance colectivo \\
\hline - Prevención prenatal & \\
- Vacunaciones de la edad & \\
infantil & - Seguridad vial \\
- Estilo de vida (consumo y & - Seguridad laboral \\
producción sanos) & - Educación para la salud \\
- Autoprevención de accidentes & - Yodación de alimentos \\
cuíficosos del envejecimiento, & - Oferta pública universal de \\
como vacuna antigripal o & vacunas \\
antineumonía & \\
\hline
\end{tabular}

Fuente: Casado (2011a: 128).

La prevención primaria debe comenzar en la fase prenatal. La iniciativa de consultar sobre posibles riesgos para el embrión y el feto es individual y voluntaria; y ocurre que está poco extendido en la población el conocimiento relativo tanto a los riesgos prenatales como a las posibilidades de su prevención La asistencia médica al embarazo es ofrecida con carácter general por el Sistema Nacional de Salud; pero no está garantizado el acceso universal a recursos especializados de gran interés, como el Servicio de Información Telefónica sobre Teratógenos (SITTE) y el Servicio Telefónico para la Embarazada (SITE) [〈http://www.fundacion100o.es〉].

La vacuna antipolio que se administra en la infancia previene deficiencias que pueden generar dependencia. La vacuna antineumónica que se recomienda en la edad avanzada tiene también efecto preventivo de secuelas que pueden suponer igualmente dependen- 
cia. Sin duda, la instrumentación de tales vacunas mediante la fórmula de campañas sanitarias logra una amplia convergencia de la oferta pública con las iniciativas individuales.

El estilo de vida sano es asunto muy personal, de modo que cabría remitir su seguimiento a la responsabilidad privada. Pero ocurre que son muchos los factores de esa deseable conducta. Veamos un ejemplo. La dieta mediterránea es un recurso muy positivo para la salud, especialmente para la etapa del envejecimiento, y que en España y países de su área dietética recibimos por herencia cultural. Pero, como es sabido, los hábitos alimentarios seculares reciben presiones de cambio. Por de pronto, estamos expuestos a la seducción de los países hegemónicos, que, desgraciadamente, tienen hábitos alimentarios menos saludables. La mercadotecnia nos incita a consumir alimentos industriales, una parte de los cuales -como la bollería de ese origen- presentan inconvenientes desde el punto de vista que aquí interesa. La educación para la salud está llamada a actuar en este frente -entre otros-. Contamos con entidades privadas comprometidas con una alimentación saludable (Fundación Dieta Mediterránea, Fundación Española de la Nutrición), pero la acción pública al respecto es muy escasa.

La prevención de accidentes laborales y de tráfico cuenta con una estimable tradición en la conciencia social y en la acción pública. Pero no cabe decir lo mismo de la prevención de otra clase de accidentes, como los domésticos y los que ocurren fuera del domicilio y no son de tráfico. La observación común muestra que incluso la población de edad avanzada no libera sus domicilios particulares de elementos de riesgo (alfombras, superficies resbaladizas, estanterías cuyo acceso exige subirse a sillas y banquetas). Gracias a la iniciativa de la Unión Europea, existe el Programa de Prevención de Lesiones, que estudia los accidentes domésticos y de ocio, pero no se observa actividad pública relevante en la acción preventiva. Cruz Roja, por su parte, realiza algunas acciones de educación para la salud relativas a la seguridad doméstica.

\subsection{Asistencia de mantenimiento}

La responsabilidad de la asistencia de mantenimiento y la provisión de ésta son asequibles, en muchos casos, a los familiares de las personas en situación de dependencia, que vienen desempeñando tradicionalmente dichos cometidos, con la colaboración de servidores domésticos o similares en ciertos supuestos. En los tiempos recientes y en nuestras sociedades, está creciendo la atención a las situaciones de dependencia por porte de agentes extrafamiliares: entidades públicas, mercantiles y privadas no lucrativas. Aparte de otros factores, la mayor incorporación de las mujeres al mercado de trabajo -y la organización uniformista de éste- están dificultando la atención familiar a la dependencia. Además del fenómeno de sustitución parcial apuntado, debe recordarse el desarrollo de medios especializados adicionales; sirvan como ejemplo los que se basan en la innovación tecnológica.

El fenómeno indicado parece haber propiciado la clasificación de los cuidados de atención a la dependencia en 'formales' -los advenidos-e 'informales' -los tradicionales-. Se obvia dicho enfoque en este artículo, por la connotación gratuitamente negativa de este segundo término (Daly y Standing, 2003: 33), el cual ha sido objeto de un amplio análisis recientemente (Sánchez Reinón, 2012).

El Cuadro 3 quiere servir para indicar, de modo sumario, la diversidad de recursos funcionales que sirven al objetivo de asistencia de mantenimiento en las situaciones de dependencia (se excluyen los monetarios, por su carácter instrumental).

Las opciones residenciales deben situarse por delante de las otras, porque: a) llevan consigo varios recursos importantes; b) influyen en la articulación de los otros recursos; $y$, además, c) son decisivas en relación a un principio fundamental en la procura del objetivo citado, el de autonomía moral.

En el Cuadro 3 se proponen dos niveles de atención. Consideramos básica la necesaria para suplir las carencias funcionales que supone la dependencia en el desempeño de las actividades de la vida diaria común: desde el cuidado personal hasta las actividades instrumentales. La asistencia especial incluye apoyos con requerimientos técnicos u organizativos que no conoce el común de la población, como la teleasistencia o la asistencia sanitaria.

En el caso del alojamiento en hogares particulares, no sólo se dispone de diversos medios para la

\begin{tabular}{|c|c|c|c|}
\hline \multirow{2}{*}{ Residencia } & \multicolumn{3}{|c|}{ Nivel de atención } \\
\hline & Básica & Básica y especial & Especial \\
\hline $\begin{array}{l}\text { Hogar particular } \\
\text { (del afectado, de su } \\
\text { familia, o de la persona o } \\
\text { familia acogedora) }\end{array}$ & $\begin{array}{l}\text { - Apoyos funcionales y relacionales } \\
\text { de familiares y otros próximos } \\
\text { - Servidores domésticos y asisten- } \\
\text { tes personales } \\
\text { - Servicio de ayuda a domicilio } \\
\text { - Comidas a domicilio }\end{array}$ & $\begin{array}{l}\text { - Teleasistencia } \\
\text { - Centro de día } \\
\text { - Centro de noche } \\
\text { - Residencia de respiro }\end{array}$ & $\begin{array}{l}\text { - Servicios sanitarios ambulatorios } \\
\text { - Hospital de día }\end{array}$ \\
\hline Establecimiento colectivo & & $\begin{array}{l}\text { - Residencia asistida } \\
\text { - Hospital de larga estancia }\end{array}$ & - Servicios sanitarios ambulatorios \\
\hline
\end{tabular}

Fuente: Elaboración propia. 
atención básica (desde los cuidados familiares hasta los servicios de ayuda a domicilio), sino que cabe hacer muchas combinaciones de éstos -salvo normas jurídicas de incompatibilidades-. Una buena parte de las personas afectas de dependencia residen en domicilios privados y conviven con familiares o, en algunos pocos casos, con personas acogedoras; en tales supuestos, es muy frecuente que reciban cuidados de aquéllos y de éstas. Algunos afectados de dependencia tienen capacidades para mantenerse en su propio hogar y reciben ayuda familiar, aunque con menos frecuencia que en las situaciones de convivencia (Rogero, 2010: 324). Por otra parte, en cualquiera de los supuestos de residencia en hogares particulares, son posibles las prestaciones de atención básica provistas por agentes externos (entidades sociales no lucrativas, empresas, organismos públicos). En estos supuestos, cabe contar también con la complementación de servicios que combinan la atención básica y la especializada: teleasistencia, centro de día y de noche, residencia de respiro. Y también es común para las situaciones de residencia en hogares particulares, la posibilidad de contar con servicios sanitarios especializados.

Las residencias de estancia continuada, sean de la rama de servicios sociales o de la sanitaria, son medios totalizantes, aunque con importantes salvedades. Por grande que sea el celo de sus gestores por la calidad asistencial, para el común de los usuarios resulta deseable el acompañamiento familiar y de voluntarios extrafamiliares que provea, mediante visitas y comunicaciones a distancia, relaciones personales genuinas. Entre otras entidades, cabe citar a Nagusilan como entidad voluntaria practicante del citado acompañamiento voluntario. Los familiares y otros próximos están llamados a jugar un papel importante en las atenciones sanitarias de los usuarios de residencias que se realizan fuera de éstas, sea de carácter ambulatorio o con ingreso en hospital de agudos. En este segundo caso, resulta muy oportuno el acompañamiento en el ingreso, durante la estancia y en el trámite de alta; no sólo para prestar apoyo afectivo, sino también para ayudar a que se tengan en cuenta las prescripciones correspondientes a sus padecimientos crónicos.

Los seguros y prestaciones monetarias pueden destinarse a financiar -o posibilitar- los diferentes medios de asistencia de mantenimiento. Algunas de las prestaciones monetarias públicas tienen regulada su aplicación a la adquisición de servicios.

\section{Dificultades para la elección de medios idóneos}

Por limitaciones de espacio, no se consideran aquí todas las clases técnicas de medios aplicables a la consecución de los diferentes objetivos concernientes a la dependencia, sino sólo las comprendidas en estos dos ámbitos: a) el de la Ley General de Sanidad (LGS) y sus normas de desarrollo, que configuran el Sistema Nacional de Salud (SNS); y b) el generado por la Ley de
Promoción de la Autonomía Personal y de Atención a las personas en situación de dependencia (LAAD).

\section{1. Ámbito de la Ley General de Sanidad}

Los medios de acción del Sistema Nacional de Salud están regulados por la Ley 16/2003, de 28 de mayo, de cohesión y calidad del Sistema Nacional de Salud, la cual establece el catálogo de prestaciones del Sistema Nacional de Salud (arts. 7-19), con estos componentes: "salud pública, atención primaria, atención especializada, atención sociosanitaria, atención de urgencias, la prestación farmacéutica, la ortoprotésica, de productos dietéticos y de transporte sanitario" (art. 7.1). Dicho catálogo fue desarrollado mediante el Real Decreto 1030/2006, de 25 de septiembre, por el que se establece la cartera de servicios comunes del Sistema Nacional de Salud.

Para la prevención sanitaria -que incluye obviamente la relativa a la dependencia-, tienen gran importancia los medios de salud pública - no confundir con la asistencia sanitaria pública-. Los comprendidos en el SNS fueron determinados por el catálogo (art. 11) y por la cartera (anexo I) indicados anteriormente. Se trata de un elenco plausible. La Ley 33/2011, de 4 de octubre, General de Salud Pública añadió algunos medios a los previstos en la Ley de cohesión y calidad, pero mantuvo los más relevantes para la prevención de la dependencia.

Por el carácter colectivo de la acciones de salud publica, su elección no se presta a la participación individual de los usuarios. En virtud de esta circunstancia, sería muy deseable que éstos participaran a través de asociaciones, como las de consumidores y usuarios, que, aparentemente, intervienen poco en esta materia. En cuanto a la prevención de la dependencia, parece que sería oportuna su acción en pro de los "programas intersectoriales y transversales de promoción y educación para la salud orientados a la mejora de los estilos de vida" previstos en la cartera de servicios del SNS (anexo I, 3.1).

La asistencia sanitaria del SNS cuenta con una amplísima gama de medios, los cuales están especificados en su cartera de servicios: de atención primaria, de atención especializada, de atención de urgencia, prestación farmacéutica, prestación ortoprotésica, productos dietéticos, transporte sanitario (anexos II a VIII). También este conjunto de medios parece plausible. Una parte de ellos son relevantes para los objetivos de prevención, rehabilitación y asistencia de la dependencia.

La elección de los medios de asistencia sanitaria se realiza mediante prescripción de facultativos, cuya condición y régimen profesional son factores propiciadores de la adecuación de sus decisiones. Por supuesto, cabe la posibilidad de errores, negligencias y conflictos de intereses, a la vez que existen mecanismos de control de las desviaciones en la práctica prescriptora. 
Parece oportuno recordar en este punto que las prescripciones no son vinculantes para el usuario, así como que tiene derecho a ser informado antes de aceptar o rechazar las que le afecten. Así lo establece la Ley 41/2002, de 14 de noviembre, básica reguladora de la autonomía del paciente y de derechos y obligaciones en materia de información y documentación clínica.

Aparte de los factores personales, en la práctica clínica ha de contarse con una circunstancia que puede llegar a impedir o a hacer inútil la elección correcta de medios: la insuficiencia de oferta. Este riesgo está conjurado -al menos jurídicamente- para todos los servicios de la cartera del SNS, el acceso a los cuales está garantizado. Se trata de una circunstancia positiva de obvia importancia, a la que, en el orden jurídico, sólo se le oponen algunos reparos de detalle; por ejemplo, la composición de la prestación ortoprotésica (anexo $\mathrm{VI}$ ) -como también, en otro plano normativo, la reglamentación del acceso-. Pero dicha cartera de servicios adolece de un grave déficit, consistente en que no incluye una de las clases de prestaciones del catálogo aprobado por la citada Ley de cohesión y calidad del Sistema Nacional de Calidad: la prestación de atención sociosanitaria (art. 14), la cual es muy relevante para la dependencia funcional.

Esa relevancia se manifiesta claramente en la regulación de la citada prestación, destinada a "enfermos, generalmente crónicos, que, por sus especiales características, pueden beneficiarse de la actuación simultánea y sinérgica de los servicios sanitarios y sociales para aumentar su autonomía, paliar sus limitaciones o sufrimientos, y facilitar su reinserción social” (art. 14.1). Incluye "cuidados sanitarios de larga duración”, "atención sanitaria de convalecencia” y "rehabilitación de pacientes con déficit funcional recuperables" (art. 14.2). Por otra parte, establece que "la continuidad del servicio será garantizada por los servicios sanitarios y sociales, a través de la adecuada coordinación entre las Administraciones públicas correspondientes" (art. 14·3).

\section{2. Ámbito de la Ley de Promoción de la Autonomía Personal y de Atención a las personas en situación de dependencia}

Como ya quedó indicado, el título del libro blanco elaborado para la preparación de la norma que vendría a ser la LAAD (Ministerio de Trabajo y Asuntos Sociales, 2005) declara sólo el objetivo de "atención a las personas en situación de dependencia”. En lo que concierne a medios, la fuente citada examinó una gama muy amplia, según muestra la siguiente reseña (no exhaustiva):

- El capítulo tercero de la obra está dedicado a "los cuidados de larga duración a cargo de la familia”.

- El capítulo siguiente, que examina los “recursos [formales] para la atención a las personas menores de 65 años en situación de depen- dencia", abarca las "prestaciones y servicios sociales, sanitarios y de la Seguridad Social para las personas menores de 65 años en situación de dependencia”. En tal examen, se incluyen los recursos relativos al objetivo de la asistencia de mantenimiento y también los correspondientes a la rehabilitación (ídem: 246, 254, 259 y 270); y en lo concerniente a la atención temprana de los "niños con limitaciones graves", se cubren todos los niveles de la prevención (ídem: 282-287).

- El capítulo quinto versa sobre los "recursos sociales para la atención a las personas mayores de 65 años", de modo que se concentra en la rama de los servicios sociales. Pero el capítulo siguiente, sobre "el sistema sanitario y la atención a las personas en situación de dependencia” abarca a todas las edades. Por otra parte, incluye expresamente los medios de prevención (ídem: 491 y 492).

Según lo expuesto anteriormente, en el anteproyecto de la LAAD y en la propia Ley, al objetivo inicial de "atención a las personas en situación de dependencia" se le antepuso el de "promoción de la autonomía personal”. Esta opción era propicia no sólo para mantener el complejo de medios que abarca el libro blanco, sino para ampliarlo. Pero veamos cuál vino a ser la presencia de lo sanitario en la LAAD.

El artículo 15 de la LAAD establece su catálogo de servicios en los siguientes términos:

1. El catálogo de servicios comprende los servicios sociales de promoción de la autonomía personal y de atención a la dependencia, en los términos que se especifican en este capítulo:

a) Los servicios de prevención de las situaciones de dependencia y los de promoción de la autonomía personal.

b) Servicio de teleasistencia.

c) Servicio de ayuda a domicilio:

(i) Atención de las necesidades del hogar.

(ii) Cuidados personales.

d) Servicio de centro de día y de noche:

(i) Centro de día para mayores.

(ii) Centro de día para menores de 65 años.

(iii) Centro de día de atención especializada.

(iv) Centro de noche.

e) Servicio de atención residencial:

(i) Residencia de personas mayores en situación de dependencia.

(ii) Centro de atención a personas en situación de dependencia, en razón de los distintos tipos de discapacidad.

2. Los servicios establecidos en el apartado 1 se regulan sin perjuicio de lo previsto en el artículo 14 de la Ley 16/2003, de 28 de mayo, de cohesión y calidad del Sistema Nacional de Salud.

Como se ve, en el apartado 1 se califica a los servicios del catálogo de “sociales", sin mención alguna de lo sanitario. Por otra parte, la salvedad que se hace en 
el apartado 2 es una derivación hacia el vacío, pues el artículo 14 de la Ley 16/2003, según quedó dicho, no se incluyó en la cartera de servicios del SNS.

El primero de los componentes del catálogo abarca "servicios de prevención", los cuales parecen llamados a incluir medios de carácter sanitario. Pero la regulación de esos servicios (art. 21) incluye una segunda disposición neutralizadora de las responsabilidades del SAAD, pues deriva la provisión a planes "que elaboren las comunidades autónomas" para tal fin.

Los "servicios de promoción de la autonomía personal", segundo componente del primer renglón del catálogo, son también propicios para la acción sanitaria, pero ocurrió que el legislador omitió regularlos en las disposiciones relativas a los servicios del catálogo de la LAAD. Este descuido contrasta con la asignación a la prestación económica de asistencia personal de la "promoción de la autonomía de las personas con gran dependencia”, como finalidad exclusiva (art. 19).

La LAAD, en la definición del servicio de centro de día y de noche, aparte de su objeto básico, le asigna la cobertura de las necesidades de "asesoramiento, prevención, rehabilitación, orientación para la promoción de la autonomía, habilitación o atención asistencial y personal" (art. 24.1). Y al servicio de atención residencial, le asigna la oferta de "servicios continuados de carácter personal y sanitario” (art. 25.1).

La cobertura de necesidades de prevención por los centros de día debería contar, al menos en el ámbito de la prescripción y la supervisión, con atención de carácter sanitario, pero no se explicita. Las prestaciones sanitarias que se asignan a las residencias de la LAAD -de la rama de los servicios sociales, según quedó indicado- parecen funcionales para los usuarios que deseen evitar desplazamientos, y pueden facilitar la coordinación y la integración de la atención social y de la sanitaria. Pero parece un descuido importante de la LAAD que no regule la responsabilidad e intervención de los servicios del SNS en la parte de esa acción mixta que le corresponde. Esa participación, por cierto, debería comenzar desde el inicio del procedimiento de acceso a la protección de la LAAD.

Las dificultades en la elección de medios, que se examina en este apartado, se plantean en las prestaciones económicas de la LAAD de modo más grave que en su catálogo de servicios. La primera circunstancia de dificultad consiste en que la ley asigna a los servicios carácter prioritario sobre las prestaciones económicas (art. 14.2). En segundo lugar, restringe el acceso a cada una de las prestaciones económica en los términos que se indican seguidamente: la "prestación económica vinculada al servicio" se reconocerá "únicamente cuando no sea posible el acceso a un servicio público o concertado de atención y cuidado" (art. 17); la "prestación económica para cuidados en el entorno familiar y apoyo a cuidadores no profesionales" es declarada excepcional (art. 18); la "prestación económica de asistencia personal" se prevé para "con- tribuir a la contratación de una asistencia personal, durante un número de horas, que facilite al beneficiario el acceso a la educación y el trabajo, así como una vida más autónoma en el ejercicio de las actividades básicas de la vida diaria” (art. 19).

La LAAD no explicita los fundamentos de las restricciones que impone a la elección de las prestaciones económicas. Tampoco salva la aparente contradicción que entrañan tales trabas frente al ideal de la autonomía personal, que es el primer objetivo de la norma (art. 1). Cabe pensar que la prioridad de los servicios sociales se estableciera en relación con los objetivos de generación de empleo y retornos económicos y sociales examinados en el capítulo $X$ del libro blanco (Ministerio de Trabajo y Asuntos Sociales, 2005), reseñados anteriormente. En fuente bien informada, se menciona el compromiso del Imserso -entidad que prestó y presta apoyo técnico a la elaboración de la LAAD y a su seguimiento- con dichos objetivos (Balbontín y Cobo, 2011: 29).

Parece oportuno indicar que, poco tiempo después de aprobarse la LAAD, se acordó un cambio legislativo favorable a la protección monetaria de una contingencia asimilable a la dependencia. La Ley General de la Seguridad Social ofrecía dos opciones al "gran inválido": pensión de invalidez incrementada en un $50 \%$ o "sustitución del incremento [...] por su alojamiento y cuidado a cargo de la Seguridad Social y en régimen de internado" (art. 139.4). La Ley 40/2007, de 4 de diciembre, de medidas en materia de Seguridad Social, establece una nueva redacción de dicho apartado en la que se suprimió la segunda posibilidad (art. 2.3).

Teniendo como referencia la autonomía de decisión, resulta oportuno echar de menos en la panoplia de la LAAD ciertas fórmulas prestacionales que hacen posible aquélla de modo seguro y que se vienen aplicando en algunos países (SIIS, 2011: 45-50). Por una parte, existen prestaciones económicas aplicables, no a un servicio o fórmula de atención dada -como establece la LAAD-, sino a la adquisición de diferentes apoyos. Y existen también figuras asistenciales consistentes en fondos individuales o personales de horas de atención, que el beneficiario consume según sus necesidades e intereses.

Para acceder a la acción protectora de la LAAD, es necesario obtener el reconocimiento de la situación de dependencia y del derecho a las prestaciones del sistema creado por aquella (art. 28). Alcanzada esa condición, se aborda el procedimiento para la elección de medios, que debe atenerse a las limitaciones señaladas. Conducen ese trámite los gestores de la LAAD y participan en él los propios interesados. Mediante esta relación, siempre que los solicitantes acepten la propuesta elaborada, se establece el programa individual de atención (PIA) [art. 29]. Ese programa sólo puede incluir medios de la panoplia de la LAAD, y, por añadidura, los gestores del PIA no sólo carecen de conexión orgánica con el SNS, sino que tampoco tienen encomendada formalmente función alguna de 
relación con él. Aparte de la eventual iniciativa autónoma de dichos gestores, las circunstancias indicadas suponen la pérdida de una oportunidad de la tan ponderada y demandada coordinación sociosanitaria.

En el plano de la aplicación, se observó en algunos territorios (Rimbau, 2010: 53-54) que, tras la aprobación de la LAAD, se produjeron restricciones en el acceso de los beneficiarios de aquélla a recursos de los servicios sociales públicos antes abiertos para las situaciones de discapacidad.

\subsection{Parches a la Ley de Dependencia}

Sin pretensión de exhaustividad, se reseñan en este apartado medidas de desarrollo y aplicación de la LAAD que palian algunas de las deficiencias señaladas en su catálogo de servicios.

El Real Decreto 727/2007, de 8 de junio, sobre criterios para determinar las intensidades de protección de los servicios y la cuantía de las prestaciones económicas de la LAAD, en su artículo 5, establece lo siguiente:

1. Las personas en situación de dependencia en alguno de los grados establecidos recibirán servicios de prevención con el objeto de prevenir el agravamiento de su grado y nivel de dependencia, incluyendo esta atención en los programas de teleasistencia, de ayuda a domicilio, de los centros de día y de atención residencial.

2. Los planes de prevención para prevenir la aparición de las situaciones de dependencia y su agravamiento, elaborados por las correspondientes comunidades autónomas o Administración que, en su caso, tenga la competencia, determinarán las intensidades de los servicios de prevención del Sistema para la Autonomía y Atención a la Dependencia en su correspondiente ámbito territorial.

Para valorar lo que dispone el primer apartado, procede notar que la LAAD no asigna la función preventiva a dos de los cuatro servicios que señala el Real Decreto: el de teleasistencia (arts. 22) y el de ayuda a domicilio (art. 23). El apartado 2 incurre también en voluntarismo y contradicción, pues se refiere a prevenir la aparición de las situaciones de dependencia, pese a que la LAAD sólo protege las situaciones ya establecidas y reconocidas (art. 5.1.a).

El artículo 6 del Real Decreto en examen, mediante sus dos primeros apartados, suple la omisión de la LAAD, antes indicada, relativa a la "promoción de la autonomía personal":

1. Los servicios de promoción de la autonomía personal tienen por finalidad desarrollary mantener la capacidad de tomar decisiones acerca de cómo vivir de acuerdo con las normas y preferencias propias, y facilitar la ejecución de las actividades básicas de la vida diaria.
2. Son servicios de promoción de la autonomía personal los de asesoramiento, orientación, asistencia y formación en tecnologías de apoyo y adaptaciones que contribuyan a facilitar la realización de actividades de la vida diaria, los de habilitación, los de terapia ocupacional, así como cualesquiera otros programas de intervención que se establezcan con la misma finalidad.

El intento de suplir la omisión de la LAAD relativa a la promoción de la autonomía personal no termina con el Real Decreto 727/2007. Éste fue modificado por la norma del mismo rango 175/2011, de 11 de febrero, que, mediante su artículo 1.3., establece para el grado I (dependencia moderada) los siguientes servicios de promoción de la autonomía personal: habilitación y terapia ocupacional; atención temprana; estimulación cognitiva; promoción, mantenimiento y recuperación de la autonomía funcional; habilitación psicosocial para personas con enfermedad mental o discapacidad intelectual; y apoyos personales y cuidados en alojamientos especiales (viviendas tuteladas).

\subsection{Contra la prestación para cuidados en el entorno familiar y apoyo a cuidadores no profesionales}

Pese a las disposiciones restrictivas concernientes a las prestaciones económicas de la LAAD antes reseñadas, la prevista "para cuidados en el entorno familiar y apoyo a cuidadores no profesionales" (art. 18) [en adelante, PECEF] fue objeto de mayor número de concesiones que cualquier otra. Según la información del Sistema de Información del Sistema para la Autonomía y la Atención a la Dependencia (SISAAD), al cabo de los tres primeros años de aplicación de la LAAD, el derecho a dicha prestación fue reconocido tantas veces como el correspondiente a los restantes medios regulados por aquélla (Tabla 1 ).

Tabla 1. Prestaciones que recibían las personas beneficiarias del Sistema para la Autonomía y la Atención a la Dependencia. España, 1 de enero de 2010

\begin{tabular}{|c|c|c|c|}
\hline \multicolumn{2}{|c|}{$\begin{array}{l}\text { Prestaciones económicas y servicios } \\
\text { reconocidos }\end{array}$} & \multirow{2}{*}{$\begin{array}{c}\text { № } \\
38.158\end{array}$} & \multirow{2}{*}{$\begin{array}{c}\begin{array}{c}\% \text { respecto a } \\
\text { concedidas }\end{array} \\
6,8\end{array}$} \\
\hline \multirow{3}{*}{$\begin{array}{l}\text { Prestaciones } \\
\text { económicas }\end{array}$} & $\begin{array}{l}\text { Prestación vinculada a } \\
\text { servicio }\end{array}$ & & \\
\hline & $\begin{array}{l}\text { Prestación para } \\
\text { cuidados en el entorno } \\
\text { familiar }\end{array}$ & 280.013 & 50,0 \\
\hline & Asistente personal & 685 & 0,1 \\
\hline \multirow{5}{*}{ Servicios } & $\begin{array}{l}\text { Prevención y promoción } \\
\text { de autonomía personal }\end{array}$ & 3.018 & 0,5 \\
\hline & Teleasistencia & 51.480 & 9,2 \\
\hline & Ayuda a domicilio & 59.576 & 10,6 \\
\hline & Centro de día/noche & 28.479 & 5,1 \\
\hline & Atención residencial & 98.941 & 17,7 \\
\hline \multicolumn{2}{|l|}{ Total } & 560.350 & 100,0 \\
\hline
\end{tabular}

Fuente: Elaboración propia a partir de datos del SISAAD (datos sin acumular). 
Teniendo en cuenta el procedimiento para la concesión de las prestaciones de la LAAD (arts. 28 y 29), parece obvio que, salvo excepciones, contó con la conformidad de las administraciones y los solicitantes. Aparte de otras circunstancias, para aquéllas la PECEF tenía la ventaja de la modestia de sus cuantías máximas. En cuanto a los solicitantes, es conocida la amplia proporción de los que desean permanecer en su domicilio y ser atendidos por sus familiares.

Las numerosas concesiones de la PECEF fueron objeto de críticas por parte de algunos observadores sin intereses particulares en el asunto, y también y de modo clamoroso por agentes privados proveedores de servicios concertables por las administraciones, según la previsión explicita de la LAAD (arts. 14.2), así como por al menos una asociación de profesionales (Casado, 2011b). En esa campaña no se valoró que la opción de los solicitantes por la PECEF es una expresión de su autonomía personal, objetivo primero de la LAAD.

La campaña contra la PECEF llegaría a verse correspondida por algunas medidas de reforma de la LAAD destinadas principalmente a reducir el gasto de su aplicación. Puede verse en otros trabajos -como el reciente de Toni Vilà (2013)- el inventario completo de tales medidas reformadoras; aquí sólo se reseñan las específicamente adversas para la PECEC.

El Consejo Territorial del SAAD, en reunión celebrada el 10 de julio de 2012, aprobó un "Acuerdo para la mejora del sistema para la autonomía personal y atención a las personas en situación de dependencia”, el cual se publicó mediante Resolución de 13 de julio de 2012, de la Secretaría de Estado de Servicios Sociales e Igualdad. Aparte de en otras cuestiones, el acuerdo, tanto en su parte diagnóstica como en la de propuestas, incide en la PECEF. Así, en las "razones que avalan las medidas de mejora propuestas", el acuerdo toma como referencia los objetivos sociales y económicos colaterales de la atención a las situaciones de dependencia y reivindica los servicios frente a la PECEF:

El incumplimiento de las expectativas generadas, tanto en empleo como en actividad económica, dado que no se ha priorizado la atención a través de la red de financiación pública de servicios sociales de las comunidades autónomas, generadora de empleo y actividad económica, primándose, por el contrario, las ayudas a cuidadores no profesionales, contraviniendo el espíritu y letra de la Ley, que lo consideraba una excepción.

En las "propuestas de mejora" se incluyen, en primer lugar, varias orientadas a ampliar los requisitos exigibles a los cuidadores familiares para el reconocimiento de la PEDEF a los afectados (segundo, $7^{\mathrm{a}}$ ). La enumeración comienza con este anuncio:

Se revisará en profundidad, conjuntamente con el Ministerio de Empleo y Seguridad Social, el actual sistema de afiliación, alta y cotización a la
Seguridad Social de los cuidadores en el entorno familiar, para establecer una nueva regulación que tenga en cuenta las circunstancias específicas de estos cuidadores dentro del sistema de Seguridad Social.

En las "medidas de ahorro y reducción del déficit público" (segundo, 15ํㅡㄹ), figura este acuerdo:

La medida de ahorro adoptada en este Consejo Territorial es reducir en un $15 \%$ las cuantías máximas de las prestaciones económicas por cuidados en el entorno familiar.

Las comunidades autónomas, en función de sus necesidades de financiación y presupuestarias y al tratarse de cuantías máximas, pueden aumentar este porcentaje de reducción, comunicándolo al Imserso a través del SISAAD.

En el apartado de "criterios y contenidos sobre capacidad económica y participación del beneficiario en el coste de las prestaciones para la autonomía y la atención a la dependencia", como para los restantes medios de la $L A A D$, se formula la regulación relativa a la PECEF (tercero, 9.2).

El Real Decreto Ley 20/2012, de 13 de julio, de medidas para garantizar la estabilidad presupuestaria y de fomento de la competitividad incluye "medidas de racionalización del sistema de dependencia" (título III). Veamos las que afectan de modo sustantivo a la PECEF.

La LAAD no reguló incompatibilidades de sus medios de acción, y algunas administraciones reconocieron combinaciones de servicios (teleasistencia y ayuda a domicilio, por ejemplo) y de prestaciones económicas con servicios (especialmente PECEF y teleasistencia 0 ayuda a domicilio). El Real Decreto Ley 20/2012 aborda esa cuestión (art. 22.9) introduciendo un nuevo artículo en la LAAD, en los siguientes términos:

Artículo 25 bis. Régimen de incompatibilidad de las prestaciones

1. Las prestaciones económicas serán incompatibles entre sí y con los servicios del catálogo establecidos en el artículo 15, salvo con los servicios de prevención de las situaciones de dependencia, de promoción de la autonomía personal y de teleasistencia.

2. Los servicios serán incompatibles entre sí, a excepción del servicio de teleasistencia, que será compatible con el servicio de prevención de las situaciones de dependencia, de promoción de la autonomía personal, de ayuda a domicilio y de centro de día y de noche.

No obstante lo anterior, las administraciones públicas competentes podrán establecer la compatibilidad entre prestaciones para apoyo, cuidados y atención que faciliten la permanencia en el domicilio a la persona en situación de dependencia, de tal forma que la suma de estas 
prestaciones no sea superior, en su conjunto, a las intensidades máximas reconocidas a su grado de dependencia. A los efectos de la asignación del nivel mínimo establecido en el artículo 9, estas prestaciones tendrán la consideración de una única prestación.

Las administraciones siguieron criterios dispares en la cuestión de la compatibilidad, de lo que se derivó desigualdad en la protección. Como quiera que la LAAD se dicta en virtud de una competencia exclusiva del Estado de fundamento igualitario (disposición final octava), parece obligado que se atajara aquella desigualdad. Pero cabría haber optado por una fórmula análoga a los fondos individuales antes reseñados, de modo que se asegurarse la igualdad en la intensidad del conjunto de las concesiones, posibilitando a la vez la adecuación del programa individual de atención (PIA) a las necesidades e intereses de los beneficiarios.

Como quedó dicho, la LAAD regula el PIA en un régimen de participación de los beneficiarios y sus familias (art. 29). El Real Decreto Ley 20/2012 establece un mecanismo de control adicional para la PECEF (art. 22.13): “la determinación de la prestación económica por cuidados en el entorno familiar corresponderá a la Administración competente, a propuesta de los servicios sociales".

La LAAD remitió a norma reglamentaria la afiliación, alta y cotización en la Seguridad Social de los cuidadores no profesionales (art. 18.3 y disposición adicional cuarta); ello se hizo mediante el Real Decreto $615 / 2007$, de 11 de mayo. Éste incluyó una disposi- ción, muy beneficiosa para dichos cuidadores, por la que asigna a la Administración General del Estado la financiación de la cotización a la Seguridad Social, así como la correspondiente a acciones formativas (art. 4.5). El Real Decreto Ley anula la aportación citada, al establecer (disposición adicional octava) que: 1) el convenio "tendrá, para los cuidadores no profesionales, carácter voluntario"; y 2) las cotizaciones a la Seguridad Social "serán a cargo exclusivamente del suscriptor del mismo”. Observación complementaria: mediante la disposición transitoria decimotercera del Real Decreto Ley, se establece el programa de liquidación de las aportaciones públicas en los conceptos indicados a los cuidadores que las estaban recibiendo en la fecha de entrada en vigor de aquél.

El Real Decreto Ley estableció las cuantías de las prestaciones económicas (disposición transitoria décima). Para facilitar la apreciación de los recortes de la PECEF, la Tabla 2 recoge las cuantías máximas vigentes en el momento de aprobarse dicha norma y las fijadas por ésta para los solicitantes que no tuvieran reconocidas prestaciones y para los nuevos solicitantes. Procede aclarar que las de 2012 sólo se refieren a los grados sin diferenciación de niveles, por haber sido suprimidos éstos por el Real Decreto Ley (art. 22.10). La ausencia de la aportación por la cotización de Seguridad Social y de formación profesional deriva de la disposición adicional octava del Real Decreto Ley, antes reseñada.

La Tabla 3 ofrece la información correspondiente a la variación de las cuantías máximas de dos prestaciones económicas que cabe considerar alternativas:

Tabla 2. Cuantías máximas mensuales de la prestación económica por cuidados en el entorno familiar. España, 2011 y 2012 (euros)

\begin{tabular}{|c|c|c|c|c|}
\hline \multicolumn{3}{|c|}{2011} & \multicolumn{2}{|c|}{2012} \\
\hline Grado y nivel & Cuantías & Cuota SS + FP & Grado & Cuantías \\
\hline III. 2 & 520,69 & \multirow{4}{*}{$\begin{array}{c}164,54 \\
(163,04+1,50)\end{array}$} & \multirow{2}{*}{ III } & \multirow{2}{*}{387,64} \\
\hline III.1 & 416,98 & & & \\
\hline II.2 & 337,25 & & \multirow{2}{*}{ II } & \multirow{2}{*}{268,79} \\
\hline II.1 & 300,90 & & & \\
\hline 1.2 & 180,00 & $\begin{array}{c}82,27 \\
(81,52+0,75)\end{array}$ & I & 153,00 \\
\hline
\end{tabular}

SS: Seguridad Social. FP: Formación Profesional.

Fuente: Elaboración propia a partir del Real Decreto 570/2011, de 20 de abril, y del Real Decreto Ley 20/2012, de 13 de julio, disposición transitoria décima.

Tabla 3. Comparación de las cuantías de la prestación económica vinculada al servicio y de la prestación económica para cuidados en el entorno familiar. España, 2011-2012 (euros)

\begin{tabular}{|c|c|c|c|c|c|c|}
\hline \multirow{3}{*}{ Año } & \multirow{3}{*}{ Grado } & \multirow{3}{*}{ Nivel } & \multicolumn{4}{|c|}{ Prestaciones } \\
\hline & & & PEVS & & PECEF & \\
\hline & & & Cuantía & Cuantía & $\begin{array}{l}\text { Cuota } \\
\text { SS + FP }\end{array}$ & Suma \\
\hline \multirow{3}{*}{2011} & III & 2 & 833,96 & 520,69 & \multirow{3}{*}{164,54} & 685,23 \\
\hline & III & 1 & 625,47 & 416,98 & & 581,52 \\
\hline & \multicolumn{2}{|c|}{ Medias } & 729,71 & 468,83 & & 633,37 \\
\hline 2012 & III & - & 715,07 & 387,64 & - & 387,64 \\
\hline \multicolumn{3}{|c|}{ Reducción para 2012 (\%) } & 2,00 & 17,32 & - & 38,79 \\
\hline
\end{tabular}

PEVS: prestación económica vinculada al servicio. PECEF: prestación económica para cuidados en el entorno familiar. SS: Seguridad Social. FP: Formación Profesional.

Fuente: Elaboración propia a partir del Real Decreto 570/2011, de 20 de abril, anexo; y del Real Decreto Ley 20/2012, de 13 de julio, disposición transitoria décima. 
la vinculada al servicio (PEVS), es decir, la destinada por la LAAD a adquirir servicios privados y no concertados (art. 17); y la PECEF. Para simplificar, la tabla incluye sólo las cuantías relativas a grado III. Por la circunstancia, ya indicada, de que el Real Decreto Ley suprimió los niveles, y para facilitar la comparación de las variaciones operadas, se calculó la media de los valores establecidos para 2011. Como puede verse, la cuantía máxima de la PEVS se reduce dos puntos porcentuales, en tanto que la reducción correspondiente a la PECEF supera en más de dos puntos porcentuales la reducción del $15 \%$ propuesta en el "Acuerdo para la mejora del Sistema para la Autonomía Personal y Atención a las Personas en Situación de Dependencia” y anteriormente reseñada. Como quiera que, después de aprobarse el Real Decreto Ley, se suspendió la subvención pública de las cuotas de la Seguridad Social y de Formación Profesional de los cuidadores, la reducción total relativa es mucho mayor.
Las medidas reseñadas muestran claramente la política disuasoria de la opción por la PECEF, anunciada en el acuerdo del Consejo Territorial en su sesión de 10 de julio de 2012. Como quiera que -con harto fundamento- se tiende a interpretar todas las reformas de la LAAD en clave de ahorro, tal vez sea oportuno señalar que las relativas a la PECEF no tienen ese objetivo, ni debido a sus bajas cuantías anteriores a la reforma producirán ese efecto en todos los casos.

¿Han surtido efecto las medidas contrarias a la PECEF? En la información de SISAAD correspondiente a enero de 2010 que aparece en la Tabla 1, los reconocimientos de dicha prestación representaban el $50 \%$ de todas las reconocidas. A 1 de agosto de 2012, fecha próxima a la de la reforma de la LAAD, ese porcentaje se había reducido a 45,3\%. En la estadística correspondiente al 30 de junio de 2013, el valor de ese indicador era de 43,6\%. ¿Mantenimiento de tendencia o cambio? 
(2012): “Real Decreto-ley 20/2012, de 13 de julio, de medidas para garantizar la estabilidad presupuestaria y de fomento de la competitividad”, Boletín Oficial del Estado, no $168,14-7-2012$, págs. 50.428-50.518 [«http://www.boe.es/diario_boe/txt. php?id=BOE-A-2012-9364'].

(2012): “Resolución de 13 de julio de 2012, de la Secretaría de Estado de Servicios Sociales e Igualdad, por la que se publica el Acuerdo del Consejo Territorial del Sistema para la Autonomía y Atención a la Dependencia para la mejora del sistema para la autonomía y atención a la dependencia, Boletín Oficial del Estado, n- 185, 3-8-2012, págs. 55.65755.674 [khttp://www.boe.es/buscar/doc. php?id=BOE-A-2012-10468〉].

(2011): “Ley 33/2011, de 4 de octubre, General de Salud Pública", Boletín Oficial del Estado, no 240, 5-10-2011, págs. 104-593-104.626 [<http://www.boe.es/diario boe/txt. php?id=BOE-A-2011-15623)].

(2011): "Real Decreto 175/2011, de 11 de febrero, por el que se modifica el Real Decreto $727 / 2007$, de 8 de junio, sobre criterios para determinar las intensidades de protección de los servicios y la cuantía de las prestaciones económicas de la Ley 39/2006, de 14 de diciembre, de Promoción de la Autonomía Personal y Atención a las personas en situación de dependencia, y el Real Decreto 615/2007, de 11 de mayo, por el que se regula la Seguridad Social de los cuidadores de las personas en situación de dependencia”, Boletín Oficial del Estado, no 42, 18-2-2011, págs. 18.69218.699 [/http://www.boe.es/buscar/doc. php?id=BOE-A-2011-3175’].
(2011): “Real Decreto 570/2011, de 20 de abril, por el que se modifica el Real Decreto $727 / 2007$, de 8 de junio, sobre criterios para determinar las intensidades de protección de los servicios y la cuantía de las prestaciones económicas de la Ley 39/2006, de 14 de diciembre, de Promoción de la Autonomía Personal y Atención a las personas en situación de dependencia y se establecen las prestaciones económicas de la Ley 39/2006, de 14 de diciembre, de Promoción de la Autonomía Personal y Atención a las personas en situación de dependencia para el ejercicio 2011", Boletín Oficial del Estado, no 112, 11-5-2011, págs. 47.93647.940 [rhttp://www.boe.es/buscar/doc. php?id=BOE-A-2011-8230>].

(2007): "Ley 40/2007, de 4 de diciembre, de medidas en materia de Seguridad Social", Boletín Oficial del Estado, no-291, 5-12-2007, págs. 50.18650.200 [shttp://www.boe.es/buscar/doc. php?id=BOE-A-2007-20910〉].

(2007): "Real Decreto 615/2007, de 11 de mayo, por el que se regula la Seguridad Social de los cuidadores de las personas en situación de dependencia”, Boletín Oficial del Estado, no -114, 12-5-2007, págs. 20.602-20.605 [रhttp://www.boe.es/ diario_boe/txt.php?id=BOE-A-2007-9690>].

(2007): “Real Decreto 727/2007, de 8 de junio, sobre criterios para determinar las intensidades de protección de los servicios y la cuantía de las prestaciones económicas de la Ley 39/2006, de 14 de diciembre, de Promoción de la Autonomía Personal y Atención a las personas en situación de dependencia”, Boletín Oficial del Estado, nํㅜ 138, de 9-6-2007, págs. 25.25625.259 [/http://www.boe.es/buscar/doc. php?id=BOE-A-2007-11446>]. 
(2006): “Ley 39/2006, de 14 de diciembre, de Promoción de la Autonomía Personal y Atención a las personas en situación de dependencia", Boletín Oficial del Estado, ํㅜ 299, 15-12-2006, págs. 44.142-44.156 [khttp://www.boe.es/boe/ dias/2006/12/15/pdfs/A44142-44156.pdf>].

(2006): “Real Decreto 1030/2006, de 15 de septiembre, por el que se establece la cartera de servicios comunes del Sistema Nacional de Salud y el procedimiento para su actualización”, Boletín Oficial del Estado, no 222, de 16-9-2006, págs. 32.650-32.679 [<http://www.boe.es/ diario_boe/txt.php?id=BOE-A-2006-16212〉].

(2003): “Ley 16/2003, de 28 de mayo, de cohesión y calidad del Sistema Nacional de Salud", Boletín Oficial del Estado, no $128,29-5-2003$, págs. 20.56720.588 [ php?id=BOE-A-2003-10715'].

(2002): “Ley 41/2002, de 14 de noviembre, básica reguladora de la autonomía del paciente y de derechos y obligaciones en materia de información y documentación clínica", Boletín Oficial del Estado, ํㅜ 274, 15-11-2002, págs. 40.126-40.132 [<http://www.boe.es/ diario_boe/txt.php?id=BOE-A-2002-22188>].

(1994): "Real Decreto Legislativo 1/1994, de 20 de junio, por el que se aprueba el texto refundido de la Ley General de la Seguridad Social”, Boletín Oficial del Estado, no 154, 29-6-1994, págs. 20.65820.708 [ http://www.boe.es/diario_boe/txt. php?id=BOE-A-1994-14960>].

(1986): “Ley 14/1986, de 25 de abril, General de Sanidad", Boletín Oficial del Estado, no-102, 29-4-1986 págs. 15.207-15.224 [shttp://www.boe.es/ diario_boe/txt.php?id=BOE-A-1986-10499'].

BALBONTÍN, A.; y COBO, P. (2011): “Acuerdos del Consejo Territorial del Sistema para la Autonomía Personal y Atención a la Dependencia sobre criterios para determinar las intensidades de protección de los servicios y las condiciones y cuantías de las prestaciones económicas", Actas de la Dependencia, $\mathrm{n}-1$, págs. 22-34 [shttp://www.fundacioncaser.es/ documentos/20110301_004'].

CASADO, D. (2011a): Afrontar la discapacidad, el envejecimiento y la dependencia, Madrid, Editorial CCS.

- (2011b): “Atención básica a la dependencia funcional”, [comunicación], III Congreso de la Red Española de Política Social, Pamplona, Universidad Pública de Navarra, 25/26-11-2011.

- (2006-2007): “La Ley de Promoción de la Autonomía Personal y Atención a las personas en situación de dependencia y contexto de medidas públicas", Documentación Administrativa, no 276-277, págs. 79-112 [rhttp://revistasonline. inap.es/index.php?journal=DA\&page $=$ article\& op=view\&path[]=9525\&path[]=9477)].

CONSEJO DE EUROPA (1999) [1998]: “Recomendación no (98) 9 del Comité de Ministros a los Estados miembros relativa a la dependencia. (Adoptada por el Comité de Ministros el 18 de septiembre de 1998 , en la 641 g reunión de Delegados de Ministros)", en IMSERSO (1999): Vejez y protección social a la dependencia en Europa. Iniciativas. Recomendaciones del Consejo de Europa, Imserso.

DALY, M.; y STANDING, G. (2003): “Introducción”, en DALY, M. (comp.), El trabajo asistencial: en busca de la seguridad, Madrid, Ministerio de Trabajo y Asuntos Sociales.

KANT, E. (1972) [1785]: Fundamentación de la metafísica de las costumbres [y otras obras], México: Editorial Porrúa.

LEICHSENRING, K.; BILLINGS, J.; y NIES H. (2013): “Improving policy and practice in long-term care", en LEICHSENRING, K.; BILLINGS, J.; y NIES H. (eds.), Long-term Care in Europe. Improving Policy and Practice, Nueva York, Palgrave MacMillan.

IMSERSO (2005): Atención a las personas en situación de dependencia en España. Libro blanco, Madrid, Imserso [khttp://www.dependencia.imserso. es/InterPresent2/groups/imserso/documents/ binario/libroblanco.pdf)].

ORGANIZACIÓN MUNDIAL DE LA SALUD (2002): "Envejecimiento activo: un marco político", Revista Española de Geriatría y Gerontología, vol. 37, supl. 2, págs. 74-103.

- (2000): Hacia un consenso internacional sobre los programas de cuidados de larga duración para las personas mayores, Organización Mundial de la Salud; Milbank Memorial Fund.

RIMBAU, C. (2010): “Impacto de la Ley de Promoción de la Autonomía Personal y de Atención a las personas en situación de dependencia en el modelo familiar de cuidado tradicional y de los servicios sociales", [tesina de máster], Universidad de Barcelona, Departamento de Teoría Sociológica, Filosofía del Derecho y Metodología de las Ciencias Sociales.

ROGERO, J. (2010): Los tiempos de cuidado. El impacto de la dependencia de los mayores en la vida cotidiana de sus cuidadores, Madrid, Imserso.

SÁNCHEZ REINÓN, M. (2012): “Los ‘cuidados informales' de larga duración en el marco de la construcción ideológica, societal y de género de los 'servicios sociales de cuidados"', Cuaderno de Relaciones Laborales, vol. 30, $\mathrm{n}$ 울, págs. 185-210.

SIIS CENTRO DE DOCUMENTACIÓN Y ESTUDIOS (2011): Prestaciones económicas y otras fórmulas de asignación económica individual en los sistemas de atención a la dependencia. Panorama comparativo internacional, DonostiaSan Sebastián, Diputación Foral de Gipuzkoa [rhttp://www.siis.net/documentos/informes/ Informeprestacioneseconomicas.pdf〉].

NACIONES UNIDAS (1983): World Programme of Action Concerning Disabled Persons, Nueva York, Naciones Unidas.

VILÀ, T. (2013): "Evolución y reforma del Sistema para la Autonomía y Atención a la Dependencia”, Actas de la Dependencia, $\mathrm{n}$ - 8, págs. 47-67. 